\title{
On a Closed Form Solution to the Constant Modulus Factorization Problem*
}

\author{
Babak Hassibi $†$ Arogyaswami Paulraj and Thomas Kailath \\ Information Systems Laboratory \\ Stanford University, Stanford CA 94305
}

\begin{abstract}
We consider the problem of separating independent constant modulus signals received by an antenna array. Assuming that the statistics of the phases of the signals are known, we derive a closed form solution for the array response vector from which the original signals can be recovered. Our method is based on estimating the higher order statisitics of the received signals, and the estimate of the array response vector is shown to be asymptotically unbiased. Simulation results are included to demonstrate the feasibility of the algorithm.
\end{abstract}

\section{Introduction}

We consider the following problem. Suppose we are given a complex $M \times N$ matrix

$$
X=\left[\begin{array}{cccc}
x_{11} & x_{12} & \ldots & x_{1 N} \\
x_{21} & x_{22} & \ldots & x_{2 N} \\
\vdots & \vdots & & \vdots \\
x_{M 1} & x_{M 2} & \ldots & x_{M N}
\end{array}\right], \quad N \gg M
$$

which we know can be factorized in the following form

$$
X=A S,
$$

where $A$ is a $M \times d$ complex matrix

$$
A=\left[\begin{array}{cccc}
a_{11} & a_{12} & \ldots & a_{1 d} \\
a_{21} & a_{22} & \ldots & a_{2 d} \\
\vdots & \vdots & & \vdots \\
a_{M 1} & a_{M 2} & \ldots & a_{N d}
\end{array}\right], \quad d \leq M
$$

and $S$ is a $d \times N$ complex matrix whose elements have unit modulus, viz.

$$
S=\left[\begin{array}{cccc}
s_{11} & s_{12} & \ldots & s_{1 N} \\
s_{21} & s_{22} & \ldots & s_{2 N} \\
\vdots & \vdots & & \vdots \\
s_{d 1} & s_{d 2} & \ldots & a_{d M}
\end{array}\right], \quad\left|s_{i j}\right|=1
$$

${ }^{\dagger}$ This research was supported by the Advanced Research Projects Agency of the Department of Defense monitored by the Air Force Office of Scientific Research under Contract F49620-93-1-0085.
Both $A$ and $S$ are assumed full rank. Note that if we write

$$
X=\left[\begin{array}{llll}
x_{1} & x_{2} & \ldots & x_{N}
\end{array}\right], S=\left[\begin{array}{llll}
s_{1} & s_{2} & \ldots & s_{N}
\end{array}\right]
$$

with

$$
x_{i}=\left[\begin{array}{c}
x_{1 i} \\
x_{2 i} \\
\vdots \\
x_{M i}
\end{array}\right] \quad, \quad s_{i}=\left[\begin{array}{c}
s_{1 i} \\
s_{2 i} \\
\vdots \\
s_{d i}
\end{array}\right]
$$

then

$$
x_{i}=A s_{i} .
$$

Therefore each component of $x_{i}$ is a linear combination of constant modulus signals. We may now state the problem we are interested in.

\section{Problem 1 (CM Factorization Problem)}

Given such an $X$, find the factorization

$$
X=A S \text {, }
$$

where $A$ and $S$ have the aforementioned properties.

An Application: To illusrate an application in which the above problem arises consider an antenna array with $M$ elements, and suppose $d$ independent FM signals are received by this antenna array. Suppose, moreover, that the array manifold is not known. Each FM signal induces an array response vector $\left[\begin{array}{llll}a_{1 i} & a_{2 i} & \ldots & a_{M i}\end{array}\right]^{T}$ (which is unknown since we have assumed the array response vector unknown), so that the total response measured at the antenna at time $i$ is

$$
x_{i}=A s_{i}, \quad i=1, \ldots, N .
$$

Therefore the factorization of Problem 1 will allow us to separate and identlify each FM signal.

Many other phase retrieval problems can be reduced to Problem 1 but we shall not consider those here.

Uniqueness: The first natural question that arises in connection to the constant modulus factorization problem is that of the uniqueness of the solution. In other words is it possible that for a given $X$,

$$
X=A_{1} S_{1}=A_{2} S_{2} \quad, \quad\left\{\begin{array}{c}
A_{1} \neq A_{2} \\
S_{1} \neq S_{2}
\end{array} ?\right.
$$


The answer to the above question has been given in [3], where under some mild conditions (which shall be satisfied by the conditions required of our development), all solutions are parametrized by

$$
A=A_{0} \Phi P \text {, }
$$

where $A_{0}$ is some solution, $\Phi$ is a diagonal matrix with unit modulus diagonal, i.e.

$$
\Phi=\left[\begin{array}{ccc}
e^{j \phi_{1}} & & \\
& \ddots & \\
& & e^{j \phi_{d}}
\end{array}\right],
$$

and $P$ is a permutation matrix that permutes the columns of $A_{0} \Phi$. Physically, this parametrization means that we may add an arbitrary phase to each of the $d$ FM signals and reorder them as well. Therefore from a physical point of view such nonuniqueness will not affect our ability to recover the signals in an unambiguous fashion.

Noisy Measurements: Another question that arises is that in practice $X$ may not exactly admit a constant modulus factorization (due to, say, the presence of noise) and all we can write is

$$
X=A S+N \text {, }
$$

for some noise matrix $N$. In this case one shall attempt to find a factorization of the form $\hat{X}=\hat{A} \hat{S}$ where $\hat{X}$ is close to $X$ in some sense.

Fo a long time, the constant modulus factorization problem was considered to be too nonlinear to admit a closed-form analytic solution, and only iterative gradient-descent schemes have been developed. These algorithms are based on the pioneering work of Godard [1] and Treichler and Agee [2], and are intimately related to alternating projection methods. These algorithms go under the name CMA's (constant modulus algorithms) and have the drawback that convergence may be slow, or that one may converge to a local minimum.

In this paper we shall use a different approach and shall show that by making assumptions on the statistics of the phases of the signals, we can find a closed-form solution to the array response matrix $A$. The method is based on estimating the higher order statistics of the received signals and can be shown to yield asymptotically unbiased estimates. The method also allows one to find the $A$ matrix in the presence of noise, and when an exact factorization does not exist. These shall be the subject of the remainder of the paper.

\section{A Closed Form Solution}

\subsection{The Solution}

Consider once more

$$
X=\underbrace{\left[\begin{array}{cccc}
a_{11} & a_{12} & \ldots & a_{1 d} \\
a_{21} & a_{22} & \cdots & a_{2 d} \\
\vdots & \vdots & & \vdots \\
a_{M 1} & a_{M 2} & \ldots & a_{M d}
\end{array}\right]}_{A} S .
$$

In this solution we shall obtain estimates of the polynomials

$$
P^{(i)}(\alpha)=\alpha^{d}+p_{1}^{(i)} \alpha^{d-1}+\ldots+p_{d}^{(i)} i=1, \ldots, M
$$

whose roots are

$$
\left\{\left|a_{i 1}\right|^{2},\left|a_{i 2}\right|^{2}, \ldots,\left|a_{i d}\right|^{2}\right\}
$$

and the polynomials

$$
P^{(i j)}(\alpha)=\alpha^{d}+p_{1}^{(i j)} \alpha^{d-1}+\ldots+p_{d}^{(i j)} i, j=1, \ldots, M
$$

whose roots are

$$
\left\{a_{i 1} a_{j 1}^{*}, a_{i 2} a_{j 2}^{*}, \ldots, a_{i d} a_{j d}^{*}\right\} .
$$

\section{Remarks:}

(1) The solution is closed form: There is no iteration involved or any problem of convergence. The $a_{i j}$ can be easily obtained by solving the polynomial equations

$$
P^{(i)}(\alpha)=0 \quad \text { and } \quad P^{(i j)}(\alpha)=0 .
$$

(2) It gives all solutions: Indeed

(a) $P^{(i)}(\alpha)=0$ gives the magnitudes of the $a_{i j}, v i z .,\left|a_{i j}\right|^{2}$. Suppose, now we choose the phases for $a_{11}, a_{12}, \ldots, a_{1 d}$. Then in view of $P^{(1 j)}(\alpha)=0$, the $a_{11} a_{j 1}^{*}, a_{12} a_{j 2}^{*}, \ldots, a_{1 d} a_{j d}^{*}$ are given, and the phases for

$$
a_{j 1}, a_{j 2}, \ldots, a_{j d} j=2, \ldots, d
$$

will be fixed. Thus each column of $A$ is unique up to an arbitrary phase.

(b) Since the $P^{(i)}(\alpha), P^{(i j)}(\alpha)$ are polynomial equations, the order of their roots may be interchanged, meaning that $A$ is arbitrary up to a permutation of its columns.

\subsection{The Assumptions}

(I) For all time instants $i$, and for all $k \neq l$, the constant modulus signals $s_{k i}$ and $s_{i i}$ are independent.

(II) We need to know

$$
E\left[s_{k i}\right]^{l}, \quad l=1,2, \ldots, d .
$$

i.e. we need to know the higher order statistics of the constant modulus signals.

The higher order statistics are readily known for the following two cases of interest:

(i) The $\left\{s_{k i}\right\}$ belong to a finite alphabet.

(ii) $s_{k i}=e^{j \phi_{k}}$, where the phases $\phi_{k i}$ are uniformly distributed over $[0,2 \pi]$. In this case

$$
E\left[e^{j \phi_{k_{l}}}\right]^{l}=0, \quad \forall l .
$$

Case (ii) is a reasonable assumption for FM signals, and is what is considered here. 


\subsection{Description of Alg. for $d=2$}

For simplicity we shall begin by describing the algorithm for the $d=2$ case. Consider

$$
x_{1 i}=a_{11} s_{1 i}+a_{12} s_{2 i},
$$

therefore

$\left|x_{1 i}\right|^{2}=\left|a_{11}\right|^{2}+\left|a_{12}\right|^{2}+a_{11} s_{1 i} a_{12}^{*} s_{2 i}^{*}+a_{11}^{*} s_{1 i}^{*} a_{12} s_{2 i}$, so that using the assumptions of the previous section

$$
E\left|x_{1 i}\right|^{2}=\left|a_{11}\right|^{2}+\left|a_{12}\right|^{2} \text {. }
$$

Likewise

$$
\begin{aligned}
\left|x_{1 i}\right|^{4}= & \left(\left|a_{11}\right|^{2}+\left|a_{12}\right|^{2}\right)^{2}+2\left|a_{11}\right|^{2}\left|a_{12}\right|^{2}+ \\
& \left(a_{11} s_{1 i} a_{12}^{*} s_{2 i}^{*}\right)^{2}+\left(a_{11}^{*} s_{1 i}^{*} a_{12} s_{2 i}\right)^{2}+ \\
& 2\left(\left|a_{11}\right|^{2}+\left|a_{12}\right|^{2}\right)\left(a_{11} s_{1 i} a_{12}^{*} s_{2 i}^{*}+a_{11}^{*} s_{1 i}^{*} a_{12} s_{2 i}\right),
\end{aligned}
$$

so that

$$
E\left|x_{1 i}\right|^{4}=\left(\left|a_{11}\right|^{2}+\left|a_{12}\right|^{2}\right)^{2}+2\left|a_{11}\right|^{2}\left|a_{12}\right|^{2} .
$$

Combining (9) and (10) we have

$$
\left\{\begin{aligned}
\left|a_{11}\right|^{2}+\left|a_{12}\right|^{2} & =E\left|x_{1 i}\right|^{2}=-p_{1}^{(1)} \\
\left|a_{11}\right|^{2}\left|a_{12}\right|^{2} & =\frac{1}{2} E\left|x_{1 i}\right|^{4}-\frac{1}{2}\left(E\left|x_{1 i}\right|^{2}\right)^{2}=p_{2}^{(1)}
\end{aligned}\right.
$$

Therefore $\left|a_{11}\right|^{2}$ and $\left|a_{12}\right|^{2}$ are roots of the following polynomial:

$$
\alpha^{2}+p_{1}^{(1)} \alpha+p_{2}^{(1)}=0 .
$$

A similar argument yields

$$
\left\{\begin{aligned}
a_{11} a_{21}^{*}+a_{12} a_{22}^{*} & =E x_{1 i} x_{2 i}^{*}=-p_{1}^{(12)} \\
\left(a_{11} a_{21}^{*}\right)\left(a_{12} a_{22}^{*}\right) & =\frac{1}{2} E\left(x_{1 i} x_{2 i}^{*}\right)^{2}-\frac{1}{2}\left(E x_{1 i} x_{2 i}^{*}\right)^{2} \\
& =p_{2}^{(12)}
\end{aligned}\right.
$$

so that $a_{11} a_{21}^{*}$ and $a_{12} a_{22}^{*}$ are roots of the following polynomial

$$
\alpha^{2}+p_{1}^{(12)} \alpha+p_{2}^{(12)}=0
$$

The general method for obtaining the polynomials $P^{(i)}(\alpha)$ and $P^{(i j)}(\alpha)$ is the same as that described above. When $d \geq 2$, we need to to compute the higher order statistics as well. The results are given below.

\subsection{The $d=3$ Case}

In this case we have

$$
x_{1 i}=a_{11} s_{1 i}+a_{12} s_{2 i}+a_{13} s_{3 i} .
$$

Computing the higher order statistics yields

$$
\begin{aligned}
E\left|x_{1 i}\right|^{2}= & \left|a_{11}\right|^{2}+\left|a_{12}\right|^{2}+\left|a_{13}\right|^{2} \\
E\left|x_{11}\right|^{4}= & \left(\left|a_{11}\right|^{2}+\left|a_{12}\right|^{2}+\left|a_{13}\right|^{2}\right)^{2}+ \\
E\left|x_{11}\right|^{6}= & \left(\left|a_{11}\right|^{2}\left|a_{12}\right|^{2}+\left|a_{11}\right|^{2}\left|a_{13}\right|^{2}+\left|a_{12}\right|^{2}+\left.\left|a_{13}\right|^{2}\right|^{3}\left|a_{13}\right|^{2}\right) \\
& 6\left(\left|a_{11}\right|^{2}+\left|a_{12}\right|^{2}+\left|a_{13}\right|^{2}\right) \times \\
& \left(\left|a_{11}\right|^{2}\left|a_{12}\right|^{2}+\left|a_{11}\right|^{2}\left|a_{13}\right|^{2}+\left|a_{12}\right|^{2}\left|a_{13}\right|^{2}\right)+ \\
& 12\left|a_{11}\right|^{2}\left|a_{12}\right|^{2}\left|a_{13}\right|^{2}
\end{aligned}
$$

from which we can obtain the coefficients of the desired polynomials as follows.

$$
\begin{aligned}
p_{1}^{(1)} & =-E\left|x_{1 i}\right|^{2} \\
p_{2}^{(1)} & =\frac{1}{2}\left[E\left|x_{1 i}\right|^{4}-\left(E\left|x_{1 i}\right|^{2}\right)^{2}\right] \\
p_{3}^{(1)} & =\frac{1}{12}\left[-E\left|x_{1 i}\right|^{6}-\left(p_{1}^{(1)}\right)^{3}-6 p_{1}^{(1)} p_{2}^{(1)}\right]
\end{aligned}
$$

The remaining polynomials are obtained in the same fashion.

\subsection{The $d=4$ Case}

In this case we have

$$
x_{1 i}=a_{11} s_{1 i}+a_{12} s_{2 i}+a_{13} s_{3 i}+a_{14} s_{4 i} .
$$

Computing the higher order statistics yields

$$
\begin{aligned}
E\left|x_{1 i}\right|^{2}= & \left|a_{11}\right|^{2}+\left|a_{12}\right|^{2}+\left|a_{13}\right|^{2}+\left|a_{14}\right|^{2}=\Sigma \alpha \\
E\left|x_{1 i}\right|^{4}= & (\Sigma \alpha)^{2}+2(\Sigma \alpha \beta) \\
E\left|x_{1 i}\right|^{6}= & (\Sigma \alpha)^{3}+6(\Sigma \alpha)(\Sigma \alpha \beta)+12(\Sigma \alpha \beta \gamma) \\
E\left|x_{1 i}\right|^{8}= & (\Sigma \alpha)^{4}+12(\Sigma \alpha)^{2}(\Sigma \alpha \beta)+48(\Sigma \alpha)(\Sigma \alpha \beta \gamma) \\
& +6(\Sigma \alpha \beta)^{2}+10368(\Sigma \alpha \beta \gamma \delta)
\end{aligned}
$$

and

$$
\left\{\begin{array}{l}
p_{1}^{(1)}=-\Sigma \alpha \\
p_{2}^{(1)}=\Sigma \alpha \beta \\
p_{3}^{(1)}=-\Sigma \alpha \beta \gamma \\
p_{4}^{(1)}=\Sigma \alpha \beta \gamma \delta
\end{array}\right.
$$

Although it is possible to derive general formulas for an arbitrary $d$, due to lack of space we shall not do so here.

\subsection{Error Analysis}

In practice the required expectations are estimated as follows

$$
\hat{E}\left|x_{1 i}\right|^{l}=\frac{1}{N} \sum_{j=1}^{N}\left|x_{1 j}\right|^{l} .
$$

It is well known that the variance of such estimates is proportional to $\frac{1}{N}$. Thus the coefficients of the estimated polynomials $\hat{P}^{(i)}(\alpha), \hat{P}^{(i j)}(\alpha)$ will have variance proportional to $\frac{1}{N}$, and it follows that the roots of these polynomials will have variance proportional to $\frac{1}{N}$.

Thus as $N \rightarrow \infty$, the variance of our estimate of $A$ tends to zeo. Thus the algorithm is asymptotically unbiased or strongly consistent.

In general, when we have estimated the polynomials, they will have some deviation from their true value. Thus,

$$
\hat{P}^{(i)}(\alpha)=P^{(i)}(\alpha)+\delta P^{(i)}(\alpha),
$$


and the root wil be perturbed from the true root $\bar{\alpha}$ to $\bar{\alpha}+\delta \alpha$. Therefore using a first order analysis, we have

$$
\delta \alpha \approx-\frac{\delta P^{(i)}(\bar{\alpha})}{\frac{d}{d \alpha} P^{(i)}(\bar{\alpha})} .
$$

The above relation shows that the computed root will be sensitive to estimation errors if $\frac{d}{d \alpha} P^{(i)}(\bar{\alpha})$ is small, i.e. if there is another root close to $\bar{\alpha}$.

\subsection{Extensions to the Case with Noise}

When we have noise our model becomes

$$
x_{i}=A s_{i}+n_{i}
$$

where $s_{i}$ is as before, and $n_{i}$ is white (spatially and temporally) noise, that is independent of $s_{i}$.

The algorithm for estimating $A$ extends in a straightforward manner, as long as we know the higher order statistics of the noise.

In the case where $n_{i j}$ is a zero mean Gaussian noise with variance $\sigma^{2}$ (as is the usual assumption) we have

$$
E\left|n_{i j}\right|^{2 l}=\prod_{k=1}^{l}(2 k-1) \sigma^{2 l}
$$

\section{Simulation Results}

In the simulations we have considered a scenario in which there are four elements in the antenna array and the number of $\mathbf{F M}$ signals is $d=4$. We have considered 4 different cases with $S N R=$ $10 d b, 20 d b, 30 d b, \infty$ (where by $S N R$ we mean the average signal-to-noise ratio over all four users). The difference between the strongest and weakest signal was on the avearge $7 d b$.

We have considered two different methods of constructing the estimates of the $a_{i j}$ from the estimated polynomials. The details of the algorithms do not concern us here. We performed 50 simulations per scenario and have computed the corresponding mean-square error for each estimated FM signal. As can be seen from the figures the mean square error decreases with the number of samples. However, it is interesting that the results do not differ substantially for $S N R=20 d b, 30 d b, \infty$.

The results indicate that the algorithm has done a good job of separating and estimating the FM signals, and may be feasible in practice. Moreover, the estimates of the $A$ matrix obtained using our method can also be used as the initial estimate in the locally convergent standard CM algorithms. Our experience shows that such initial estimates invariably led to convergence of the CM algorithm within a few iterations.
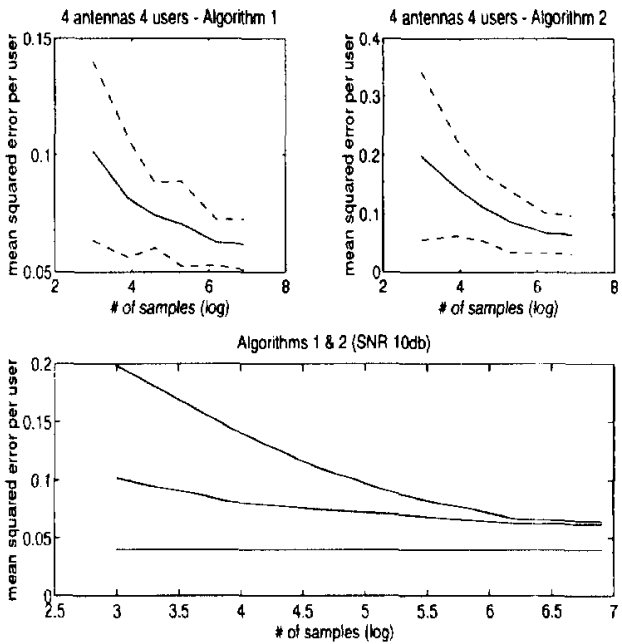

Figure 1: Results for $S N R=10 \mathrm{db}$. Dashed lines represent standard deviation. The number of samples ranged from 20 to 500 .
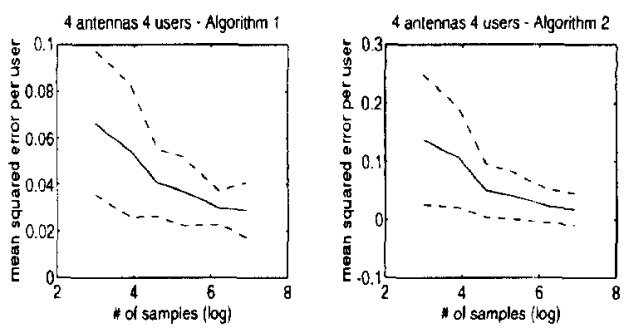

Algorithms 1 \& 2 (SNR 20db)

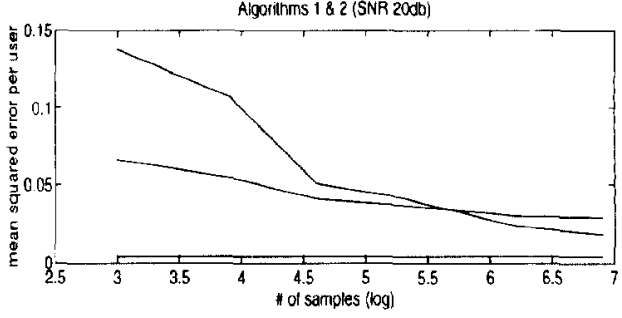

Figure 2: Results for $S N R=20 d b$. Dashed lines represent standard deviation. The number of samples ranged from 20 to 500 . 

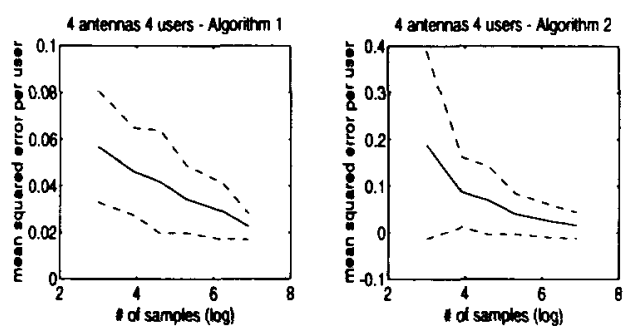

Agorithms 182 (SNA 3000)

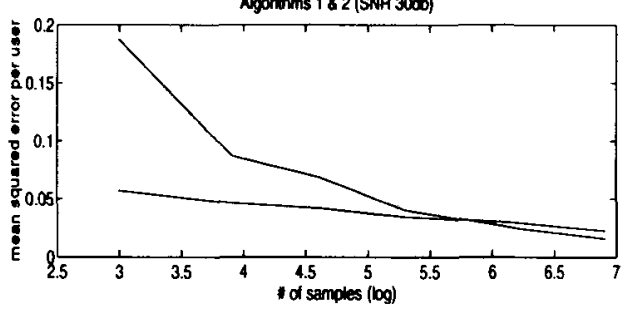

Figure 3: Results for $S N R=30 d b$. Dashed lines represent standard deviation. The number of samples ranged from 20 to 500 .
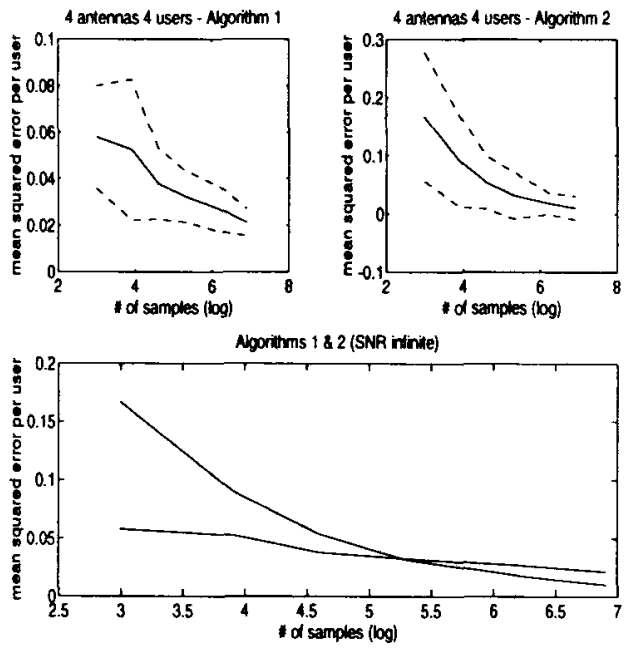

Figure 4: Results for $S N R=\infty$. Dashed lines represent standard deviation. The number of samples ranged from 20 to 500 .
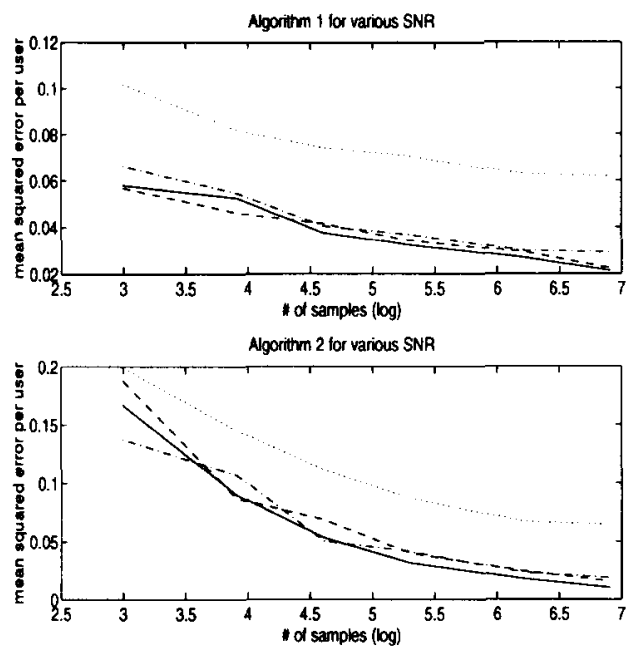

Figure 5: Comparison of different $S N R$ values. The number of samples ranged from 20 to 500 .

\section{Conclusion}

A closed form solution to the constant modulus problem was developed, and an algorithm proposed based on this solution. The proposed algorithm avoids iteration and convergence problems, and was shown to be asymptotically unbiased. The feasibility of the algorithm was demonstrated using simple simulations.

For future work more simulation is required to compare the algorithm with the standard CMA algorithms. It also would be interesting to generalize the algorithm to include for frequency selective (or FIR) channels. Although this was not done here, the algorithm can be made recursive.

\section{References}

[1] D. N. Godard Self-recovering equalization and carrier tracking in two-dimensional data and communaication systems, IEEE Trans. on Communications, vol. 28, pp. 1867-1875, Nov 1980 .

[2] J. R. Treichler and B. G. Agee A new approach to multipath correction of constant modulus signals, IEEE Trans. on ASSP, vol. 31, pp. 456471, Apr. 1983.

[3] Y. Wang, Y. C. Pati, A. Paulraj and T. Kailath A matrix factorization approach to a signal copy of constant modulus signals arriving at an antenna array, in Proc. CISS, (Princeton). 1994. 